\title{
Short Pulse Transmission Characteristics in Multi-Contact SOA
}

\author{
Kevin Carney, Robert Lennox*, Regan Watts, Severine Philippe, \\ Louise Bradley*, Pascal Landais, Senior Member, IEEE \\ The Rince Institute, School of Electronic Engineering, Dublin City University, Dublin 9, Ireland \\ * School of Physics, Trinity College Dublin, Dublin 2, Ireland \\ Tel: (+353) 01 7005884,e-mail: kevin.carney2@mail.dcu.ie
}

\begin{abstract}
An experimental characterisation of a multi-contact semiconductor optical amplifier using ultrashort optical pulses is presented. The SOA in question allows the injection of bias current through multiple independent electrical contacts, allowing the direct control of the carrier density. Picosecond-scale optical pulses are transmitted through the SOA. The non-linear effects on the pulse shape and spectrum after transmission are determined. It is found that the bias current distribution in the SOA is a significant factor in determining the extent of the non-linearities affecting the pulses. Additionally, amplification of negatively chirped pulses in the saturated SOA is found to reduce the spectral width of the pulses.
\end{abstract}

Keywords: Semiconductor optical amplifier, carrier density, ultrashort pulses, non-linearities.

\section{INTRODUCTION}

The use of ultrashort optical pulses is a critical aspect of optical communications schemes as bit rates reach $40 \mathrm{~Gb} / \mathrm{s}$ and beyond. At speeds over $100 \mathrm{~Gb} / \mathrm{s}$, pulse widths on the order of a few picoseconds are necessary in order to avoid inter-symbol interference. The narrow pulse widths attainable through the use of mode locked laser diodes makes this possible [1] and allows efficient utilization of the available bandwidth.

Semiconductor optical amplifiers (SOAs) are suitable devices for use in pulse amplification schemes due to their wide amplification bandwidth $(>5 \mathrm{THz})$. This allows them to amplify pulses on the order of a few picoseconds in duration without distortion due to gain dispersion and other effects [2]. The penalizing effect of dispersion that affects ultrashort pulses in long stretches of fibre does not have a significant effect in SOAs due to the short length of the devices, however non-linear phenomena such as gain saturation and self phase modulation can cause pulse distortions, depending on the pulse energy injected into the SOA. An optical pulse with energy below the saturation energy of the SOA is generally amplified with few distortions, but non-linear effects come into play once the pulse energy reaches this limit.

The SOA that is presented in this work is designed to either reduce noise figure or increase saturation power, depending on the distribution of carrier density within the device. This device has been previously characterised [3]. Control over this distribution is accomplished through the use of multiple electrical contacts for the injection of bias current. In this way, multiple carrier density profiles can be created depending on the required use of the SOA. The SOA presented herein has three electrical contacts, although the number of contacts is limited only by practical considerations. In order to decrease the SOA noise figure, the bias current is increased at the input of the SOA, in order to increase population inversion at the beginning of the chain and thus reduce NF for the entire device [4]. Conversely, in order to increase saturation power, the carrier density must be increased at the output of the SOA. This is due to the inverse dependence of the saturation intensity on the spontaneous carrier lifetime, $\tau_{s}$, which decreases with increasing bias current. As the signal is amplified, it encounters a steadily increasing carrier density, keeping the SOA in linear operation.

\section{NON-LINEAR EFFECTS ON ULTRASHORT PULSES IN SOAS}

When the input power to the SOA is high enough, the gain saturates, due to the bleaching of carriers by stimulated emission. Gain saturation in SOAs can give rise to a number of non-linearities that affect both the temporal and spectral characteristics of optical pulses. These non-linearities have the ability to significantly impede data transmission.

\subsection{Temporal effects}

The instantaneous gain $G$ of an SOA in response to an optical pulse is shown in equation (1).

$$
G(\tau)=\frac{G_{0}}{G_{0}-\left(G_{0}-1\right) \exp \left(-E(\tau) / E_{\text {sat }}\right)}
$$

where $G_{0}$ is the unsaturated SOA gain, $E(\tau)$ is the energy of the pulse integrated to time $\tau$, and $E_{s a t}$ is the pulse energy at which the SOA saturates. When a pulse propagates in an SOA, the leading edge of the pulse will experience the maximum unsaturated gain $G_{0}$. The corollary of this result is that the trailing edge of the pulse experiences the least amount of amplification, due to the saturation of the gain by the leading edge. In this case $E(\tau)$ is replaced in equation (1) by $E_{i n}$, the total input energy of the pulse. This saturation temporally broadens the 
pulse, as the reduced gain experienced by the trailing edge causes it to "stretch out" [5]. In addition to this, the leading edge of the pulse is sharpened. The peak of the pulse is also shifted to earlier times with increasing pulse energy, due to the saturation of the gain occurring earlier for a given pulse energy.

\subsection{Spectral effects}

Dynamic carrier density variations in SOAs not only cause a change in the gain characteristics, but also lead to a phase variation in the SOA. A change in the carrier density results in a corresponding change in the imaginary component of the complex refractive index. This in turn is accompanied by a change in the real component, the extent of which is governed by the linewidth enhancement factor $\alpha$ [6],

$$
\alpha=-\frac{4 \pi}{\lambda} \frac{d n_{e} / d N}{d g / d N}
$$

where $\lambda$ is wavelength, $n_{e}$ is the effective refractive index, $g$ is the material gain and $N$ is carrier density. This causes a change in phase. The variation of phase with gain means that an optical pulse can modulate its own phase through self phase modulation (SPM). SPM can distort pulses through frequency chirping, which is the variation of the instantaneous frequency across the profile of the pulse. The overall effect of SPM is to asymmetrically shift the pulse spectrum to longer wavelengths, often with the formation of multiple peaks [7]. SPM also leads to a broadening of the pulse spectrum, for initially unchirped and positively chirped pulses, and spectral compression if the pulses are negatively chirped [8].

\section{EXPERIMENT}

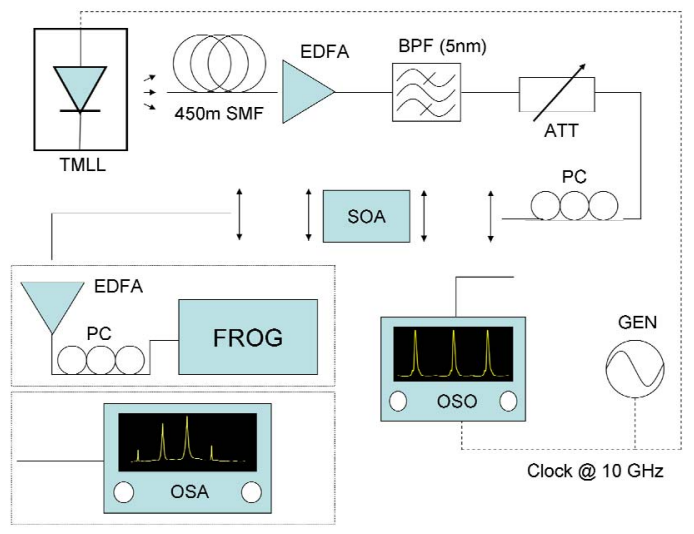

Figure 1. Diagram of experimental setup.

The experimental setup is shown in Fig. 1. The pulses are generated by a tuneable mode locked laser diode (TMLL) at a wavelength of $1550 \mathrm{~nm}$ and have a pulse width of approximately $1.5 \mathrm{ps}$. The TMLL has a saturable absorber section that enables passive mode locking within a range of 9.8 to $10.8 \mathrm{GHz}$. A $10 \mathrm{GHz}$ clock from a signal generator is applied to the TMLL to enable hybrid mode locking. The clock is also used to trigger an optical sampling oscilloscope (OSO) in order to confirm the mode locking of the laser. The TMLL is biased at $\sim 50 \mathrm{~mA}$, which is slightly above the turn-off current. The reason for this is because at higher biases, satellite pulses can appear next to the main pulse peak.

The bias current is supplied to the SOA in three configurations. In the standard configuration, $50 \mathrm{~mA}$ is injected into each contact, approximating a single contact SOA. In the low noise configuration, $90 \mathrm{~mA}$ is injected at the input contact, $50 \mathrm{~mA}$ in the middle and $10 \mathrm{~mA}$ at the output. The high saturation power configuration is the inverse of this case. For all of these configurations the total bias is set at $150 \mathrm{~mA}$, resulting in the same overall value of the carrier density in the SOA. It should also be noted that the contact sections of the SOA are not exactly the same length, and therefore the standard bias configuration is not exactly equivalent to a uniformly biased single section SOA.

The temporal profile of the pulses is measured using second harmonic generation Frequency Resolved Optical Gating (SHG-FROG) [9]. This technique allows complete characterisation of the pulse shape and phase. In order to account for time ambiguity in the FROG traces, it is necessary to introduce a known phase shift. The pulses are passed through $450 \mathrm{~m}$ of single mode fibre (SMF). The anomalous group velocity dispersion in the SMF introduces a negative chirp on the pulses, and also causes the temporal width to broaden to $\sim 4.3 \mathrm{ps}$. The pulses then are amplified with an EDFA before being passed through a wide band optical filter (5 $\mathrm{nm})$, a variable attenuator and a polarisation controller. Pulses are injected into the SOA using lenses with a numerical aperture of 0.68. At the output of the SOA, an EDFA amplifies the pulses so that the optical power is at a suitable level for efficient second harmonic generation. A polarisation controller is also necessary as the FROG is polarisation 
sensitive. The temporal and spectral resolution of the FROG is $26.66 \mathrm{fs}$ and $0.05 \mathrm{~nm}$, respectively. In addition to the FROG, the spectral characteristics of the pulses are measured using an optical spectrum analyzer (OSA) with a resolution of $0.02 \mathrm{~nm}$.

\section{RESULTS}

The temporal profiles of the pulses at various energies, measured at the output of the SOA using the FROG, are shown in Fig. 2a. The pulse energy was varied from $1 \mathrm{fJ}$ to $400 \mathrm{fJ}$. Also plotted for comparison is the profile of the pulse before injection. The sharpening of the leading edge and the stretching of the trailing edge is apparent, as is the shift of the peak towards the leading edge. Figure $2 b$ shows the measured pulse widths at the SOA output at various energies, for each of the three bias configurations. The pulse before injection is also plotted. It is interesting to note that the reduced gain saturation enabled by the high $P_{\text {sat }}$ configuration reduces the effect of pulse broadening, indicating that this bias configuration extends the linear range of operation of the SOA. The greatest broadening is seen for the standard configuration, indicative of the higher unsaturated gain of this mode.
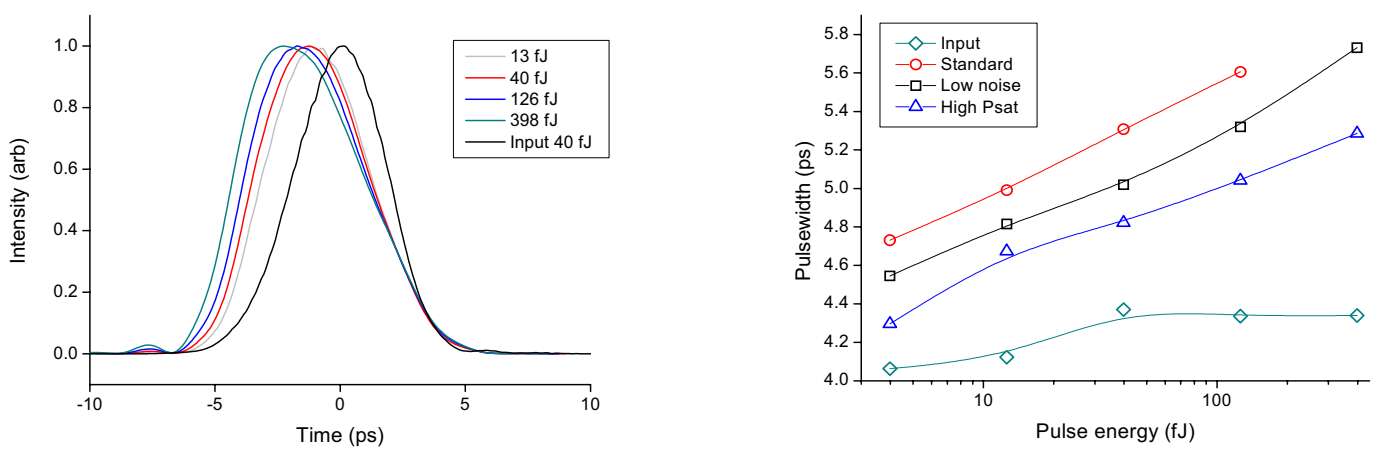

Figure 2a. Temporal profiles of pulses at SOA output, for various input pulse energy. SOA is in standard bias configuration. Figure 2b. Measured pulse widths at SOA output for various pulse energies and SOA bias configurations.

The optical spectrum of the pulse before and after injection into the SOA is shown in Fig. 3a below. The pulse energy for this figure is $398 \mathrm{fJ}$, with the SOA bias current set to the standard configuration. The main feature of the plot is the redshift of the spectrum of the pulse at the SOA output relative to the pulse before the SOA. The change in the peak wavelength of the pulse for this pulse energy is measured as $\sim 0.45 \mathrm{~nm}$. A multi-peak structure is also visible in the spectrum of the output pulse, due to interference effects. Both of these results are due to self phase modulation in the SOA brought about by gain saturation. Another notable feature of the optical spectra shown in Fig. 3a is that the spectral width of the output pulse is narrower than that of the input pulse. This is a direct consequence of SPM, combined with the negative chirp of the pulse before injection into the SOA. The standard bias configuration shows a more significant effect, with a reduction in spectral width of up to $25 \mathrm{GHz}$ at input pulse energy of $398 \mathrm{fJ}$. The smallest effect is seen in the high $P_{\text {sat }}$ bias configuration, due to the weaker SPM process in this case. These results are shown in Fig. 3b. It is expected that for initially up-chirped pulses, the resultant spectral broadening would be least detrimental in the high $P_{\text {sat }}$ bias configuration.
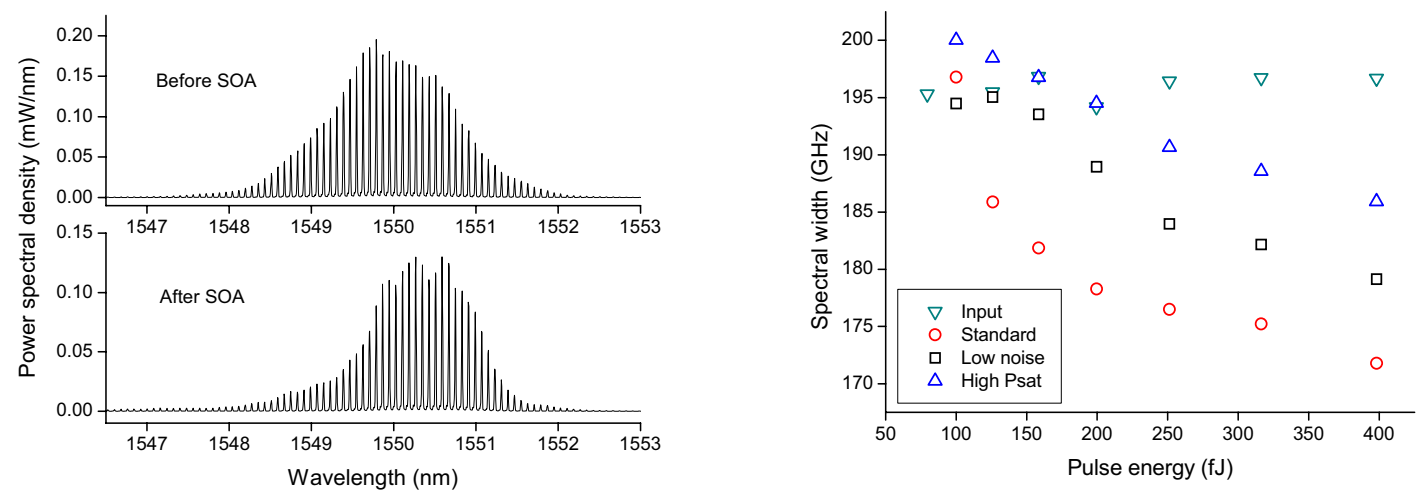

Figure 3a. Optical spectra of 398 fJ pulse before and after injection in SOA, bias in standard configuration. Data is taken from OSA measurements. Figure $3 b$. Spectral width of pulses at output of SOA as a function of input pulse energy. Spectral width of pulses at input of SOA also plotted for comparison. 


\section{CONCLUSIONS}

The effect of the SOA carrier density distribution on the non-linear effects of pulse propagation is clear. The broadening of the pulse width associated with gain saturation in SOAs is minimized when the SOA is operating in the high $P_{\text {sat }}$ mode, due to the additional linear range afforded by this carrier density distribution. Interestingly, it is the standard bias current distribution, approximating a single contact SOA, exhibits the most broadening. This result suggests that such a device may find application as a power booster for pulsed laser diodes in communications systems. The results of the spectral characterisation show a similar trend, with the greatest reduction in spectral width occurring for the standard case, while the spectral width for the high $P_{\text {sat }}$ case experienced a much less severe reduction. Since these pulses were negatively chirped, it is expected that, in the case of positively chirped optical pulses, the standard bias configuration would suffer significant temporal and spectral broadening compared with both the low noise and high $P_{\text {sat }}$ bias configurations.

\section{ACKNOWLEDGEMENTS}

The authors would like to thank Enterprise Ireland for its support under the Project CFTD/06/IT/332 and the Higher Education Authority via the INSPIRE Programme.

\section{REFERENCES}

[1] S. Latkowski et al:: Sub-picosecond pulse generation by 40-GHz passively mode-locked quantum-dash 1mm-long Fabry-Pérot laser diode, Optics Express, vol.17, no. 21, pp.19166-19172, Oct. 2009.

[2] G.P Agrawal: Fiber Optic Communications Systems, John Wiley \& Sons, 1997.

[3] R. Lennox et al.: Impact of bias current distribution on the noise figure and saturation power of a multicontact semiconductor optical amplifier, Optics Letters, vol. 36, no. 13, pp. 2521-2523, July 2011.

[4] K. Carney et al.: Noise controlled semiconductor optical amplifier based on lateral cavity laser, Electronics Letters, vol. 46, no. 18, pp. 1288-1289, Sept. 2010.

[5] J.M. Tang et al:: The influence of gain compression on picosecond optical pulses in semiconductor optical amplifiers, Journal of Modern Optics, vol. 45, no. 6, 1998.

[6] C. Henry: Theory of the linewidth of semiconductor lasers, IEEE Journal of Quantum Electronics, vol. 18, no. 2, pp. 259-264, Feb. 1982.

[7] G.P Agrawal, N.A. Olsson: Self-phase modulation and spectral broadening of optical pulses in semiconductor laser amplifiers, IEEE Journal of Quantum Electronics, vol. 25, no. 11, pp. 2297-2306, Nov. 1989.

[8] T. Watanabe et al:: Chirp control of an optical signal using phase modulation in a semiconductor optical amplifier, IEEE Photonics Technology Letters, vol. 10, no. 7, pp. 1027-1029, Jul. 1998.

[9] R. Trebino, D.J. Kane: Using phase retrieval to measure the intensity and phase of ultrashort pulses: Frequency-resolved optical gating, Journal of the Optical Society of America A, vol. 10, no. 5, pp. 11011111, 1993. 